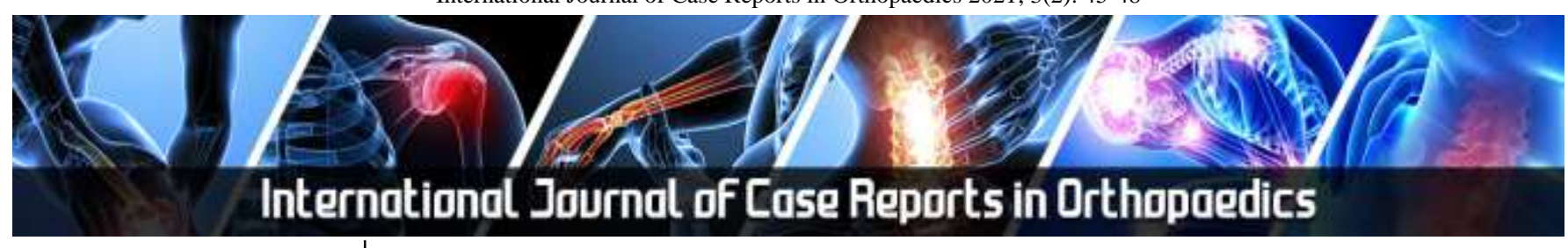

E-ISSN: 2707-8353 P-ISSN: 2707-8345 IJCRO 2021; 3(2): 45-48 Received: 24-05-2021 Accepted: 26-06-2021

Nafiu Olatunde Aigoro FWACS Consultant Orthopaedic Surgeon, Department of Orthopaedics and Traumatology State Hospital, Abeokuta, Nigeria

Ufuoma Ogigirigi MWACP, Senior Registrar, (Family Medicine) Department of surgery Sacred Heart Hospital Lantoro, Abeokuta, Nigeria

Corresponding Author: Nafiu Olatunde Aigoro FWACS Consultant Orthopaedic Surgeon, Department of Orthopaedics and Traumatology State Hospital, Abeokuta, Nigeria

\section{Floating elbow, an uncommon clinical entity: A case report}

\section{Nafiu Olatunde Aigoro and Ufuoma Ogigirigi}

DOI: https://doi.org/10.22271/27078345.2021.v3.i2a.67

\begin{abstract}
Floating elbow is an uncommon complex injury pattern with varied functional outcomes. The term is used to describe combined fractures of humerus and forearm bones in the same extremity. It could occur in children and adults. We describe a 49 years old right - handed male farmer who fell off a moving motorbike into a ditch, sustaining a closed injury to the right upper extremity. Radiographs showed right humeral shaft fracture, comminuted fracture of radial head, fracture of proximal $3^{\text {rd }}$ of Ulna and dislocation of the $5^{\text {th }}$ Metacarpo-phalangeal joint. He had plate and screw fixation of both humerus and ulna fractures, $\mathrm{K}$ wire fixation of $5^{\text {th }} \mathrm{MP}$ joint and excision of radial head. All fractures united but he has residual stiffness of the fingers and limited forearm rotation 6 months postoperatively. No Neurological sequel.
\end{abstract}

Keywords: Floating Elbow, Polytrauma, multiple fractures

\section{Introduction}

Floating Elbow injuries are rare, complex and serious lesion characterized by the fractures of the humerus and one or both bones of ipsilateral forearm ${ }^{[1,2.3]}$. The associated soft tissue injuries and involvement of the articular surface of the elbow joint make the outcome worse in the variants than the Classic or traditional floating elbow ${ }^{[4,5,6]}$.

We hereby report a case of a patient who sustained closed fracture of distal humerus and ipsilateral fracture of proximal ulna, fracture head of radius and dislocation of the $5^{\text {th }}$ Metacarpophalangeal joint following a road crash.

\section{Case Report}

A 49 years old right-handed previously healthy male farmer who was involved in a road crash as a passenger on a motorbike that ran into pot-hole while on top-speed. He landed on his right upper limb, thus sustaining closed injury on the upper arm, elbow and forearm, and a laceration on the right palm. He also sustained bruises on his right leg. There was no Loss of consciousness. The rider, who sustained minor bruises was said to have fled the scene with his motorbike.

He was taken to a near-by private clinic where he received initial treatment with suturing of the palmar laceration and application of an improvised splint to the upper limb. He was received at the emergency room of our facility about 10 hours after the incident.

Physical examination revealed swelling of the right upper arm, deformed elbow and forearm and a $4 \mathrm{~cm}$ sutured laceration over the hypothenar eminence. His Blood Pressure was 130/80mmHg. No neurological deficit.

Plain Radiographs of the right humerus, Elbow, forearm and hand showed comminuted fracture of the distal third of humerus, displaced fracture the right proximal ulna, displaced fracture of radial head and dislocation of $5^{\text {th }}$ Metacarpophalangeal joint.

He was placed on Intravenous fluids, and blood samples taken for laboratory investigations Full blood count, Hepatitis B surface Antigen, electrolytes, serum urea and serum creatinine, Retroviral screening. Analgesics and broad spectrum antibiotics exhibited.

The Patient's condition was optimized with his elevated blood pressure controlled with oral Nifedipine $30 \mathrm{mg}$ daily and Tab lexotan $1.5 \mathrm{mg}$ nocte.

48 hours after admission, the Patient underwent open reduction and internal fixation of both the humeral shaft and Ulna shaft fracture, excision of radial head and Pin fixation of $5^{\text {th }} \mathrm{MP}$ joint. Preoperatively, it was decided to fix the humeral fracture before attending to the Ulna fracture. 
Post operatively an above elbow POP backslab was applied which was removed 3 weeks after the operation. The post operative Check $\mathrm{x}$ rays were satisfactory. Patient was continued on antibiotics for 5 days and also his antihypertensive medications. All sutures were removed 14 days post-operation. $\mathrm{He}$ was discharged home to be followed up at the Out-patient clinic. Gentle elbow, and finger exercises were commenced on outpatient basis

The patient was reviewed regularly at the surgical outpatient clinic with x-rays taken at 6 weeks, 12 weeks and 6 months post op, and was adjudged to be making progress as the osteosyntheses were healing satisfactorily. However, finger stiffness persisted probably for lack of consistency in attending physiotherapy.

\section{Discussion}

The Classic Floating Elbow injuries and variants are uncommon injuries that have been reported in both children and Adults ${ }^{[1,2,3]}$. Stanistiki and Micheli ${ }^{[3]}$ in 1980 first used the term "floating Elbow" to describe the injury pattern of ipsilateral supracondylar humerus and forearm fractures that create an unstable intermediate articulation in children. The term has been extended to describe similar injury pattern in adults. The classic or traditional floating elbow is seen as a different entity to variant injuries which may have different injury mechanisms and poorer clinical outcomes due to direct involvement of the elbow joint ${ }^{[4,5]}$.

In 2013, Ditsios et al. [4] came up with a prognostic classification for adult with floating elbow injury, which is as follows:

- Group/type I - Humeral shaft fracture + radius and/or ulna shaft fracture

- Group/type IIa - Humeral shaft fracture + intra-articular radial head and/or olecranon fracture

- Group/type IIb - Intra-articular distal humerus fracture + radius and/or ulna shaft fracture

- Group/type III - Intra-articular distal humerus fracture + intra-articular radial head and/or olecranon fracture

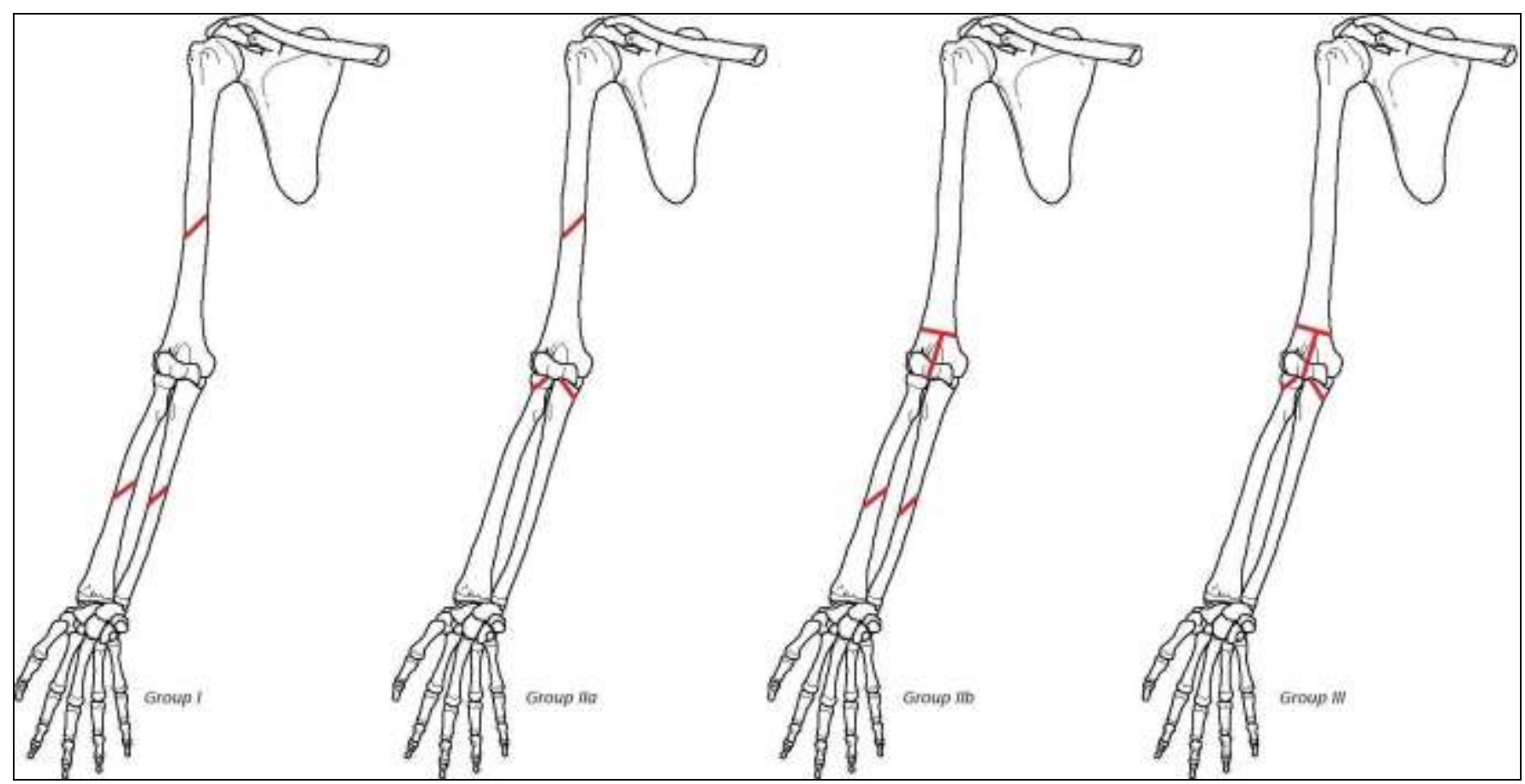

Ditsios Floating Elbow Prognostic Classification ${ }^{[6]}$.

Functional outcome is significantly poor with intra-articular involvement. Also outcomes are poor with associated neurologic injury and non-operative management ${ }^{[7,8]}$.

The rationale for treatment options of isolated humeral shaft fractures, isolated forearm fractures are of necessity different when these fractures are combined in same extremity. It has been shown that fixation of these humerus /ulna/radius fractures do better than non- operative/ conservative management.

Various forms of devices - internal and external - have been used in the treatment of this complex injury. Commonly percutaneous pins, plate and screws, intramedullary Pin/nails and external fixator are used as necessary. The operative treatment may be carried out as one- stage or two- staged procedure ${ }^{[2,6]}$.

Our patient had Ditsios Type IIa Floating elbow, and expectedly was treated operatively. In our case, Locked plate and screws were used for both the humeral and ulna shaft fractures, in a one-staged procedure.

Complications are rife in floating elbow variants than in classic/traditional type as documented in the literature ${ }^{[4,8,9}$, 10].

Yokoyama et al. ${ }^{[8]}$ in their report of 15 floating elbow in 14 patients concluded that these injuries have complications such as infection, non-union, and neurovascular injury with potential for long term functional disability.

Chul-Hyun Cho et al. ${ }^{[1]}$ in their case series of 6 patients, reported 2 patients with elbow joint stiffness with incomplete recovery from nerve injury and 1 case of complex regional pain syndrome.

Although extremely rare, Floating Elbow injury in association with floating shoulder had also being reported in a high-energy trauma in the literature ${ }^{[9]}$. 

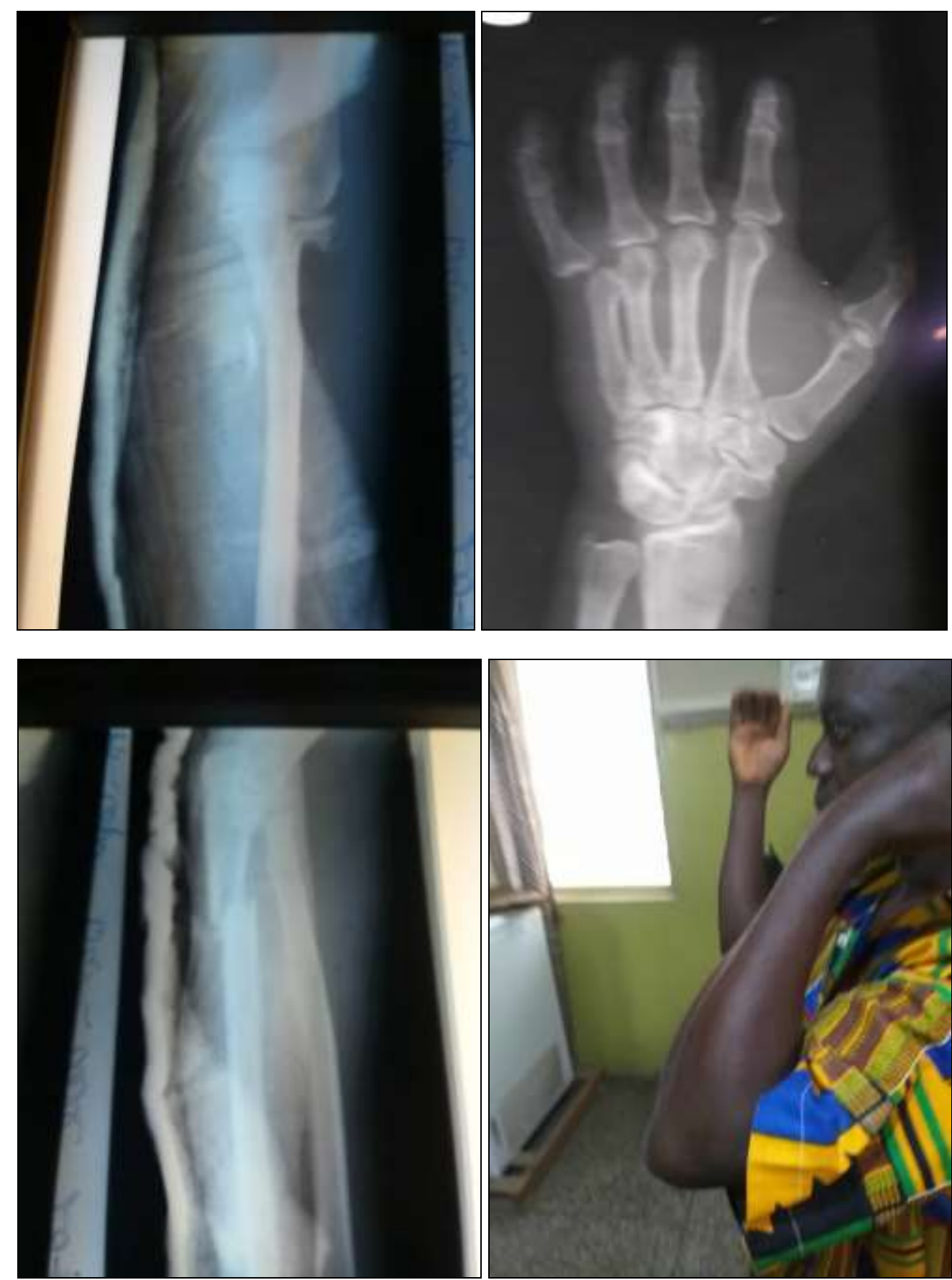

Fig 1a, 1b, 1c, 1d: Radiographs of the Right Upper arm, elbow, and Hand at Presentation
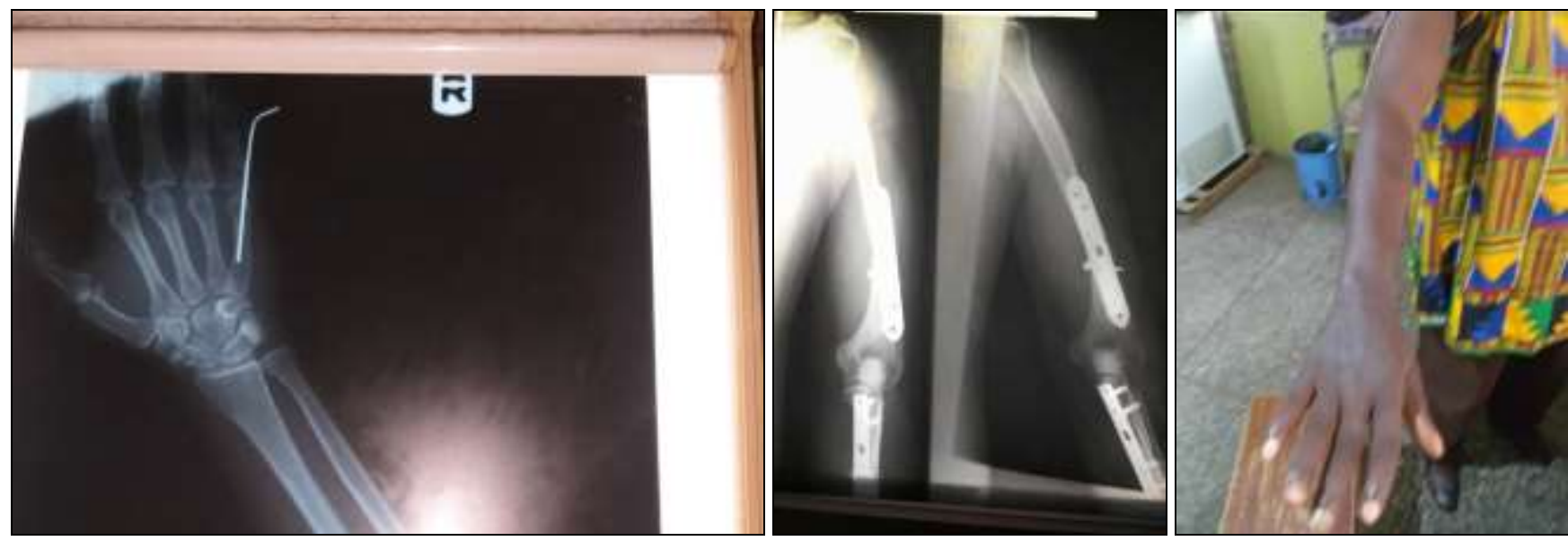

Fig 2a, 2b, 2c: Post-operative X Rays of the Right Humerus, forearm, and Hand 

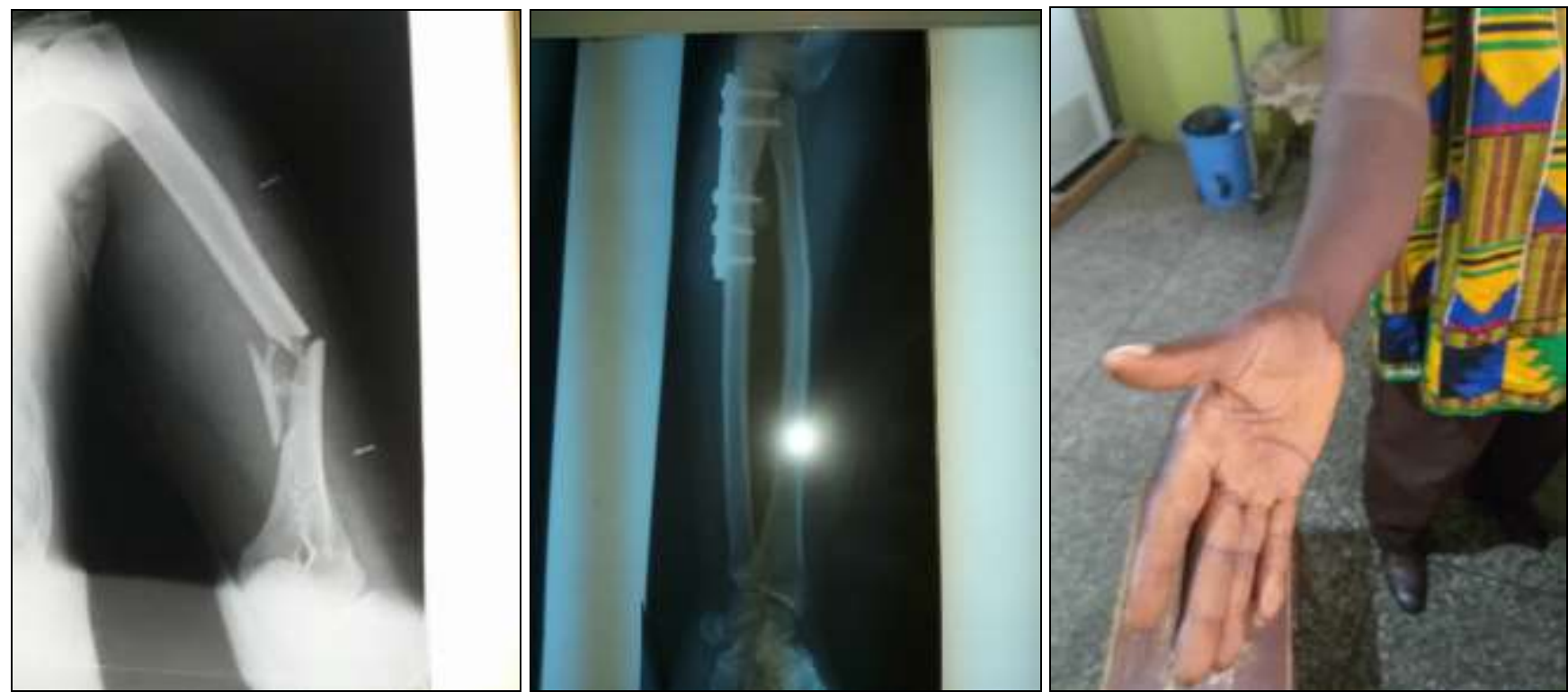

Fig 3a, 3b, 3c: Range of motion in elbow and forearm

\section{Conclusion}

Floating Elbow injuries - Classic and its variants - are uncommon but complex injuries with potential for longterm functional disability. They require well- thought out plan of management and focused rehabilitation for optimal functional outcome.

\section{Source of Support: Nil.}

\section{Conflict of Interest: None}

\section{References}

1. Chul-Hyun Cho, Kyung-Keun Min. Classic Floating Elbow in Adults. Clinics in Shoulder and Elbow 2015;18(1).

2. Suresh SS. Management of "floating elbow" in Children. Indian Journal of Orthopaedics 2007;41(4):386-389

3. Stanitsk CL, MIchelli LJ. Simultaneous ipsilateral Fracture of the Arm. Orthop Related Research 1980;(153):218-22.

4. Ditsios K, Boutsiads A, Papadopoulos P, Karataglis D, Givissis P, Hatzokos I, et al. Floating elbow injuries in adults: prognostic factors affecting clinical outcomes. Journal Shoulder Elbow Surgery 2013;22(1):74-80.

5. Lee P, Piatek AZ, DeRogatis MJ, Issack PS. Combined Ipsilateral Humeral Shaft and Galeazzi Fractures Creating a Floating Elbow Variant. Case Reports in Orthopaedics. Volume 2018, Article ID 7430297.

6. William Oros. Floating Elbow. emedicine.medscape.com. Accessed $24^{\text {th }}$ July 2021.

7. Nicola Bellona. Floating Elbow in a Polytrauma Patient: Timing and Surgical Strategy. Injury 2015;46(7):520 -522.

8. Yokoyama K, Itoman M, Kobayashi A, Shindo M, Futami T. Functional outcomes of "floating elbow" injuries in adult patients. J orthopaedic Trauma 1998;12(4):284-290.

9. El Hacen S, Zakaria R, Charafeddine EK, Mohammed R, Abdelhak G. The Association of a Floating Elbow and a Homolateral Dislocated Floating Shoulder (About a Case). European Journal of Medical and Health Sciences 2021;3(1).

10. Marius M, Olivier OET, Didace MM, Albert NO, Armand M. Floating Elbows in Adults: epidemiology, clinical presentation, management and prognosis: Brazzaville Teaching Hospital Experience. Int $\mathbf{J}$ Res Orthop 2020;6(2):323-326. 